\title{
ON THE STRUCTURE OF GENERALIZED POLYHEDRAL SEMIGROUPS WITH ZERO DEFICIENCY
}

\author{
K. AHMADIDELIR, H. DOOSTIE AND R. GHOLAMI
}

\begin{abstract}
The polyhedral or triangle groups and some generalizations of them, such as binary polyhedral groups, have been studied by several authors. In this paper, we introduce two classes of semigroups that have the same presentation as these generalizations of polyhedral groups and investigate their structures, such as finiteness, their relationship with the groups presented by the same presentation.
\end{abstract}

\section{Introduction}

The polyhedral groups $(l, m, n)$, for $l, m, n>1$ are defined by the presentation

$$
\left\langle a, b, c \mid a^{l}=b^{m}=c^{n}=a b c=1\right\rangle,
$$

or

$$
\left\langle a, b \mid a^{l}=b^{m}=(a b)^{n}=1\right\rangle
$$

and are classic in group theory. They are important both in algebra and [4] geometry. It is known that the polyhedral group $(l, m, n)$ is finite if and only if $\frac{1}{l}+\frac{1}{m}+\frac{1}{n}>1$, and in this case the order of them is obtained by the formula

$$
\frac{2 l m n}{m n+n l+l m-l m n} \text {. }
$$

For more details see $[7,8,9,11]$.

Threlfall (1932) considered the larger group $\langle l, m, n\rangle$ defined by the [4] presentation:

$$
\left\langle a, b, c \mid a^{l}=b^{m}=c^{n}=a b c\right\rangle,
$$

and called them the binary polyhedral groups. Since $(l, m, n)$ occurs as a factor group, $\langle l, m, n\rangle$ is infinite when $\frac{1}{l}+\frac{1}{m}+\frac{1}{n} \leqslant 1$. If $\left.\frac{1}{l}+\frac{1}{m}+\frac{1}{n}\right\rangle 1$, it can be shown that, the order of $\langle l, m, n\rangle$ is twice that of $(l, m, n)$, namely $\frac{4 l m n}{m n+n l+l m-l m n}$. For more information on these groups see [9].

Corresponding author: K. Ahmadidelir.

2000 Mathematics Subject Classification. 20M05, 20M10, 20M12, $20 \mathrm{~F} 05$.

Key words and phrases. Polyhedral groups, presentation of algebraic structures (groups, [4] semigroups and monoids). 
Another generalization of polyhedral groups are defined by the presentation

$$
\left\langle a, b \mid a^{l}=b^{m}=(a b)^{n}\right\rangle,
$$

which is the generalization of the second presentation of $(l, m, n)$. By adjoining a new generator $t=a b$ to this presentation we have:

$$
\left\langle a, b, t \mid a^{l}=b^{m}=t^{n}, a b=t\right\rangle .
$$

Now, if the new relation $t^{n}=1$ is adjoined then we obtain a new group presented by it, which is isomorphic to a factor group presented by (4):

$$
\left\langle a, b, t \mid a^{l}=b^{m}=t^{n}=1, a b t^{-1}=1\right\rangle .
$$

But, by setting $t^{-1}=c$ we have:

$$
\left\langle a, b, c \mid a^{l}=b^{m}=c^{n}=a b c=1\right\rangle .
$$

The groups presented by the latter presentation is isomorphic to the groups presented by the former presentation by the Tietze Transformations. So, the groups presented by (4) have the polyhedral groups as factor groups of them, and therefore are infinite if $\frac{1}{l}+\frac{1}{m}+\frac{1}{n} \leqslant 1$.

Now, consider the presentation

$$
\left\langle a, b \mid a^{l}=b^{m},(a b)^{n}=1\right\rangle .
$$

The groups presented by this presentation are the Miller's generalization of the polyhedral groups having them for a factor group [9].

In this paper we consider the semigroups presented by the presentations (4) and (6), and investigate their structure and their relationship with the groups presented by (4) and (6).

To be more precise, let:

$$
\pi_{1}=\left\langle a, b \mid a^{l}=b^{m}=(a b)^{n}\right\rangle,
$$

and

$$
\pi_{2}=\left\langle a, b \mid a^{l}=b^{m}, a(a b)^{n}=a\right\rangle .
$$

Our notation is standard and, following $[1,2,9,14]$, we recall the notion of presentation $\langle X \mid R\rangle$ of formal generators $X$ and relators $R$ where $\langle X \mid R\rangle$ is defined appropriately for finitely generated semigroups and for finitely generated monoids. For more information on group, semigroup and monoid presentations and the related algorithms one may consult $[1,2,3,12$, $13,14]$. 
To avoid confusion we denote a semigroup presentation by $S g(\pi)$, a monoid presentation by $\operatorname{Mon}(\pi)$ and a group presentation by $G p(\pi)$. Note that we will refer to $[1,2,3,4,5,6,12,13$, 14] for certain results which are necessary for our calculations.

Our main results in this paper are the following theorems:

Theorem A. $S g\left(\pi_{1}\right)$ is finite if and only if $l \leqslant 2, m \leqslant 2$. If $l=1$ or $m=1$, then $S g\left(\pi_{1}\right)$ is monogenic. But if $l=m=2$, then it has a minimal two-sided ideal $I$ such that $I \cong G p\left(\pi_{1}\right)$, and so

$$
\left|S g\left(\pi_{1}\right)\right|=\left|G p\left(\pi_{1}\right)\right|+4 n=4 n^{2} .
$$

Theorem B. For every $l, m, n \geqslant 2,\left|S g\left(\pi_{2}\right)\right|=\left|G p\left(\pi_{2}\right)\right|+(m-1)$. So $\operatorname{Sg}\left(\pi_{2}\right)$ is finite if and only if $G p\left(\pi_{2}\right)$ is finite.

Some preliminaries are necessary. For an alphabet $A$ let $A^{+}$be the free [4] semigroup over $A$, and let $A^{*}=A^{+} \cup \epsilon$ be the free monoid over $A$. For a subset $R$ of $A^{+} \times A^{+}$(respectively of $A^{*} \times A^{*}$ ) let $\rho$ be a congruence relation generated by $R$, then the semigroup $S=A^{+} / \rho$ (the monoid $M=A^{*} / \rho$ ) will be denoted by $\langle A \mid R\rangle$ which is called a semigroup presentation for $S$ (a monoid presentation for $M$ ). To lessen the likelihood of confusion, for $\omega_{1}, \omega_{2} \in A^{+}$we write $\omega_{1} \equiv \omega_{2}$ if $\omega_{1}$ and $\omega_{2}$ are identical words, and $\omega_{1}=\omega_{2}$ if they represent the same element of $S$ (i.e. if $\left.\left(\omega_{1}, \omega_{2}\right) \in \rho\right)$. Thus, for example, if $A=\{a, b\}$ and $R$ is $\{a b=b a\}$, then $a b a=a^{2} b$ but $a b a \not \equiv a^{2} b$.

Proposition 1.1. Let $\pi=\langle X \mid R\rangle$ be a presentation. If $\operatorname{Sg}(\pi)$ is finite then, $G p(\pi)$ is finite too (So, $S g(\pi)$ is infinite if $G p(\pi)$ is infinite).

Proof. Let $\pi=\langle X \mid R\rangle$, where $X$ is a non-empty finite set and $R$ a collection of relations $\alpha=\beta$ with $\alpha$ and $\beta$ non-empty words over $X$. Let $G=G p(\pi)$ and $S=S g(\pi)$. If $S$ is finite then, for each $x \in X$, there exist $i>j$ with $x^{i}=x^{j}$ in $S$, and then $x^{i-j}=1$ in $G$; so every element of $X$ has finite order in $G$, giving that $G$ is a homomorphic image of $S$, and so $G$ is finite. Thus, if $G$ is infinite, then $S$ must be infinite.

Proposition 1.2. Let $G=G p\left(\pi_{1}\right)$. Then $\frac{G}{G^{\prime}}$ is infinite if and only if

$$
\frac{1}{n}=\frac{1}{l}+\frac{1}{m}
$$

Also, $z=a^{l}$ has infinite order when $(\star)$ holds, and so $Z(G)$ is infinite in this case. 
Proof. $\frac{G}{G^{\prime}}$ has the presentation:

$$
\begin{aligned}
& \left\langle a, b \mid a^{l}=b^{m}=(a b)^{n},[a, b]=1\right\rangle \\
= & \left\langle a, b \mid a^{l} b^{-m}=1, a^{n} b^{n-m}=1, a b=b a\right\rangle .
\end{aligned}
$$

So the relation matrix of $\frac{G}{G^{\prime}}$ is:

$$
\left[\begin{array}{cc}
l & -m \\
n & n-m
\end{array}\right] .
$$

Now, $\frac{G}{G^{\prime}}$ is infinite if and only if the determinant of this matrix is equal to zero, i.e., $l(n-m)-$ $(-m) n=0$, or, $\frac{1}{n}=\frac{1}{l}+\frac{1}{m}$.

Now, when $(\star)$ holds, by exhibiting a suitable homomorphism onto $\mathbb{Z}$, we can show that $z=a^{l}$ has infinite order. But $\left\langle a^{l}\right\rangle$ is a central subgroup of $G$. So, $Z(G)$ is infinite.

Proposition 1.3. Let $G p\left(\pi_{1}\right)=[l, m, n]$. Then the only finite cases of $[l, m, n]$ are:

\section{Table 1}

\begin{tabular}{|c|c|}
\hline$[\boldsymbol{l , m}, \boldsymbol{n}]$ & Order \\
\hline$[1, m, n]$ & $m n+n-m$ \\
\hline$[l, 1, n]$ & $\ln +n-l$ \\
\hline$[2,2, n],(n>1)$ & $4 n(n-1)$ \\
\hline$[2, n, 2] \cong[n, 2,2]$ & $4 n$ \\
\hline$[2,3,2] \cong[3,2,2]$ & 12 \\
\hline$[2,3,3] \cong[3,2,3]$ & 72 \\
\hline$[3,4,2] \cong[4,3,2]$ & 48 \\
\hline$[2,4,3] \cong[4,2,3]$ & 240 \\
\hline$[2,3,4] \cong[3,2,4]$ & 336 \\
\hline$[3,5,2] \cong[5,3,2]$ & 120 \\
\hline$[2,5,3] \cong[5,2,3]$ & 320 \\
\hline$[2,3,5] \cong[3,2,5]$ & 2280 \\
\hline
\end{tabular}

Proof. By a theorem of Wiegold in [15],

$$
G=\left\langle a, b \mid a^{l}=b^{m}=\omega(a, b)\right\rangle,
$$

(where $\omega(a, b)$ is a word in terms of generators $a$ and $b$ ) is finite if and only if $\frac{G}{G^{\prime}}$ and $\frac{G}{\left\langle a^{\prime}\right\rangle}=$ $\left\langle a, b \mid a^{l}=b^{m}=\omega(a, b)=1\right\rangle$ are both finite. So, $G p\left(\pi_{1}\right)=[l, m, n]$ is finite if and only if $\frac{G}{G^{\prime}}$ and $H=\left\langle a, b \mid a^{l}=b^{m}=(a b)^{n}=1\right\rangle$ are both finite. But by Proposition 1.2, $\frac{G}{G^{\prime}}$ is finite if and only if $\frac{1}{n} \neq \frac{1}{l}+\frac{1}{m}$ and $H$ is finite if and only if $\frac{1}{l}+\frac{1}{m}+\frac{1}{n}>1$. So, the above cases are the only cases in which the group $G p\left(\pi_{1}\right)=[l, m, n]$ is finite. 
Now, since $H=(l, m, n)$ is the polyhedral group and the finite cases of $H$ are:

$$
\begin{aligned}
& (1, m, n) \cong C_{|n-m|},(2,2, n) \cong D_{2 n}, \\
& (2,3,3) \cong A_{4}, \quad(2,3,4) \cong S_{4}, \quad \text { and } \quad(2,3,5) \cong A_{5},
\end{aligned}
$$

and since

$$
(l, m, n) \cong(m, n, l) \cong(n, m, l)
$$

we have

$$
\frac{[2,2, n]}{\left\langle a^{2}\right\rangle}=\frac{[2,2, n]}{\left\langle b^{2}\right\rangle} \cong(2,2, n) \cong D_{2 n} .
$$

On the other hand $|a|=4(n-1)$ in $[2,2, n]$ and so $\left|a^{2}\right|=2(n-1)$. Therefore, $|[2,2, n]|=4 n(n-1)$. The order of other cases can be calculated easily.

Some important facts about the groups presented by $\pi_{2}$ are gathered in the following proposition (For more information about these groups one may see [9, pp.71-76]):

Proposition 1.4. Let $\left.G p\left(\pi_{2}\right)=\langle l, m \mid n\rangle, \quad(l, m, n\rangle 1\right)$ (If at least one of the $l, m$, or $n$ is equal to 1 then we have the degenerate case and $\langle l, m \mid n\rangle$ is cyclic). Then we have the followings:

(i) $\langle l, m \mid n\rangle$ is finite if and only if $k=l m+l n+m n-l m n>0$,

(ii) $|\langle l, m \mid n\rangle|=|(l, m, n)| \cdot o\left(a^{l}\right)=|(l, m, n)| \cdot o\left(b^{m}\right)$,

(iii) If $k>0$, then $o\left(a^{l}\right)=o\left(b^{m}\right)=\frac{2(l+m) n}{k}$, and $s o|\langle l, m \mid n\rangle|=\frac{4(l+m) l m n^{2}}{k^{2}}$,

(iv) $Z(\langle l, m \mid n\rangle)=\left\langle a^{l}\right\rangle=\left\langle b^{m}\right\rangle$, and so the centre is cyclic of order $\frac{2(l+m) n}{k}$,

(v) $\langle l, m \mid n\rangle \cong\langle m, l \mid n\rangle$,

(vi) The only cases that $\langle l, m \mid n\rangle$ is finite, are: 
Table 2

\begin{tabular}{|c|c|c|}
\hline$\langle l, m \mid n\rangle$ & Isomorphic to & \multicolumn{2}{|c|}{ Order } \\
\hline$\langle 2,2 \mid n\rangle$ & $\langle 2,2, n\rangle \times C_{n} \quad(n$ odd $)$ & $2 n^{2} \quad(n \in \mathbb{N})$ \\
\hline$\langle 2, m \mid 2\rangle \cong\langle m, 2 \mid 2\rangle$ & $D_{m} \times C_{m+2} \quad(m$ odd $)$ & $2 m(m+2) \quad(m \in \mathbb{N})$ \\
\hline$\langle 3,3 \mid 2\rangle$ & & 96 \\
\hline$\langle 3,2 \mid 3\rangle \cong\langle 2,3 \mid 3\rangle$ & $\langle 2,3,3\rangle \times C_{5}$ & 120 \\
\hline$\langle 4,3 \mid 2\rangle \cong\langle 3,4 \mid 2\rangle$ & & 336 \\
\hline$\langle 2,4 \mid 3\rangle \cong\langle 4,2 \mid 3\rangle$ & $\langle 2,3,4\rangle \times C_{9}$ & 432 \\
\hline$\langle 2,3 \mid 4\rangle \cong\langle 3,2 \mid 4\rangle$ & & 480 \\
\hline$\langle 3,5 \mid 2\rangle \cong\langle 5,3 \mid 2\rangle$ & & 1920 \\
\hline$\langle 2,5 \mid 3\rangle \cong\langle 5,2 \mid 3\rangle$ & $\langle 2,3,5\rangle \times C_{21}$ & 2520 \\
\hline$\langle 2,3 \mid 5\rangle \cong\langle 3,2 \mid 5\rangle$ & $\langle 2,3,5\rangle \times C_{25}$ & 3000 \\
\hline
\end{tabular}

Proof. See [9, pp.71-76].

\section{Proof of Theorem A}

First we prove a lemma:

Lemma 2.1. In the semigroup $S$, where $S=S g\left(\pi_{1}\right)$ and $l=m=2$, the element $a^{4(n-1)}$ is an idempotent of S.

Proof. Let $S=S g\left(\pi_{1}\right)$ and $l=m=2$. Then by the first and second relations of $\pi_{1}$ we get the following equations consecutively:

$$
\begin{aligned}
a^{4(n-1)+3} & =a \cdot\left(a^{2}\right)^{2 n-1} \\
& =a \cdot b^{2} \cdot\left(a^{2}\right)^{2 n-2)} \\
& =a \cdot b \cdot a^{2} \cdot b \cdot\left(a^{2}\right)^{2 n-3} \\
& =a \cdot b \cdot a \cdot b^{2} \cdot a \cdot b \cdot\left(a^{2}\right)^{2 n-4} \\
& =a \cdot b \cdot a \cdot b \cdot a^{2} \cdot b \cdot a \cdot b \cdot\left(a^{2}\right)^{2 n-5} \\
& =a \cdot b \cdot a \cdot b \cdot a \cdot b^{2} \cdot a \cdot b \cdot a \cdot b \cdot\left(a^{2}\right)^{2 n-6} \\
& =a \cdot b \cdot a \cdot b \cdot a \cdot b \cdot a^{2} \cdot b \cdot a \cdot b \cdot a \cdot b \cdot\left(a^{2}\right)^{2 n-7} \\
& =\vdots \quad \vdots \quad \vdots \\
& =\underbrace{(a b)(a b) \cdots(a b)} \cdot \underbrace{(b a)(b a) \cdots(b a)} \cdot b(n \text { times } a b, n-1 \text { times } b a) \\
& =(a b)^{n} \cdot(b a)^{n-1} \cdot b \\
& =a^{2} \cdot(b a)^{n-1} \cdot b
\end{aligned}
$$




$$
=a \cdot(a b)^{n}=a \cdot a^{2}=a^{3}
$$

Now, we have:

$$
\begin{aligned}
a^{4(n-1)} \cdot a^{4(n-1)} & =a^{4(n-1)} \cdot a^{3} \cdot a^{4(n-1)-3} \\
& =a^{4(n-1)+3} \cdot a^{4(n-1)-3} \\
& =a^{3} \cdot a^{4(n-1)-3} \\
& =a^{4(n-1)} .
\end{aligned}
$$

Proof of Theorem A. Let $S=S g\left(\pi_{1}\right), G=G p\left(\pi_{1}\right)$. If $m>2$ or $l>2$, then by Proposition 1.3, $G$ is infinite except from the cases in Table 1, so by Proposition 1.1, $S$ is infinite for the same values of $l, m$ and $n$. Now, if $l=2$ and $m=3$, then none of the relations in $\pi_{1}$ can be applied to a word of the form $\left(a b^{2}\right)^{k},(k \in \mathbb{N})$, and hence the elements:

$$
a b^{2},\left(a b^{2}\right)^{2},\left(a b^{2}\right)^{3}, \ldots
$$

are all distinct in $S$, and so it is infinite. The same is true for the cases $l=2, m=4$, with words of the form $\left(a b^{3}\right)^{k} ; l=2, m=5$, with words of the form $\left(a b^{4}\right)^{k} ; l=2, m=5$, with words of the form $\left(a b^{4}\right)^{k} ; l=3, m=4$, with words of the form $\left(a^{2} b^{3}\right)^{k}$; and $l=3, m=5$, with words of the form $\left(a^{2} b^{4}\right)^{k},(k \in \mathbb{N})$. Also if we substitute the values of $l$ and $m$ in the above cases we obtain anti-isomorphic semigroups with the corresponding semigroups, and so the remaining cases in the Table 1 are also infinite. Obviously, if $l=1$ or $m=1$, then $G$ and also $S$ are cyclic.

Finally, let $l=m=2$. First we show that $S$ has a unique minimal left ideal and a unique minimal right ideal. We claim that $S^{1} a^{3}$ is the unique minimal left ideal and $a^{3} S^{1}$ is the unique minimal right ideal of $M$. We have to show that:

$$
\begin{aligned}
& \forall \omega \in\{a, b\}^{+}, \exists \omega_{1} \in\{a, b\}^{+} ; \omega_{1} \omega=a^{3}(1), \\
& \forall \omega \in\{a, b\}^{+}, \exists \omega_{1} \in\{a, b\}^{+} ; a^{3}=\omega \omega_{1} \text { (2). }
\end{aligned}
$$

The proof of (1) is by induction on $|\omega|$ (the length of the word $\omega$ ). If $|\omega|=1$ then $\omega \equiv a$ or $\omega \equiv b$. If $\omega \equiv a$, we set $\omega_{1} \equiv a^{2}$ and then, $\omega_{1} \omega=a^{2} \cdot a=a^{3}$. If $\omega \equiv b$, we put $\omega_{1} \equiv a b$ and then, $\omega_{1} \omega=a b \cdot b=a \cdot b^{2}=a \cdot a^{2}=a^{3}$.

Now, suppose that the assertion holds for all $\omega$ such that $|\omega|<k+1[4](k \in \mathbb{N})$, and let $|\omega|=k+1$. If $\omega \equiv \omega^{\prime} a$, since $\left|\omega^{\prime}\right|=k<k+1$, then by the induction hypothesis we get:

$$
\exists \omega_{1}^{\prime} \in\{a, b\}^{+} ; \quad \omega_{1}^{\prime} \omega^{\prime}=a^{3} .
$$

Letting $\omega_{1} \equiv a^{4(n-1)-1} \omega_{1}^{\prime}$ gives us (by Lemma 2.1):

$$
\omega_{1} \omega=a^{4(n-1)-1} \underbrace{\omega_{1}^{\prime} \cdot \omega^{\prime}} a=a^{4(n-1)-1} \cdot a^{3} \cdot a=a^{4(n-1)+3}=a^{3} .
$$


If $\omega \equiv \omega^{\prime \prime} b$, since $\left|\omega^{\prime \prime}\right|=k<k+1$, then

$$
\exists \omega_{1}^{\prime \prime} \in\{a, b\}^{+} ; \quad \omega_{1}^{\prime \prime} \omega^{\prime \prime}=a^{3} .
$$

So, by considering $\omega_{1} \equiv a^{4(n-1)-3} b a \omega_{1}^{\prime \prime}$ we deduce that (again by Lemma 2.1):

$$
\begin{aligned}
\omega_{1} \omega & =a^{4(n-1)-3} b a \underbrace{\omega_{1}^{\prime \prime} \cdot \omega^{\prime \prime}}_{1} b=a^{4(n-1)-3} b a \cdot a^{3} \cdot b \\
= & =a^{4(n-1)-3} b a^{4} b \\
& =a^{4(n-1)-3} a^{4} b^{2}=a^{3} .
\end{aligned}
$$

This completes the proof of (1), showing that $S^{1} a^{3}$ is the unique minimal left ideal of $S$.

To prove (2) we use a similar method. If $|\omega|=1$, then $\omega \equiv a$ or $\omega \equiv b$. If $\omega \equiv a$, we set $\omega_{1} \equiv a^{2}$ and we get the identity $\omega \omega_{1}=a \cdot a^{2}=a^{3}$. If $\omega \equiv b$, we set $\omega_{1} \equiv b a$. Then, $\omega \omega_{1}=b \cdot b a=$ $b^{2} \cdot a=a^{3}$.

Letting $\omega=a \omega^{\prime}$ where $|\omega|=k+1$ and $\left|\omega^{\prime}\right|=k$ yields

$$
\exists \omega_{1}^{\prime} \in\{a, b\}^{+} ; \quad \omega^{\prime} \omega_{1}^{\prime}=a^{3} .
$$

So, by defining $\omega_{1} \equiv \omega_{1}^{\prime} a^{4(n-1)-1}$, we get:

$$
\omega \omega_{1}=a \underbrace{\omega^{\prime} \cdot \omega_{1}^{\prime}} a^{4(n-1)-1}=a \cdot a^{3} \cdot a^{4(n-1)-1}=a^{4(n-1)+3}=a^{3} .
$$

In the case $\omega \equiv b \omega^{\prime \prime}$ where $\left|\omega^{\prime \prime}\right|=k<k+1$, the same argument gives us

$$
\exists \omega_{1}^{\prime \prime} \in\{a, b\}^{+} ; \quad \omega^{\prime \prime} \omega_{1}^{\prime \prime}=a^{3},
$$

and, by taking $\omega_{1} \equiv \omega_{1}^{\prime \prime} a b a^{4(n-1)-3}$, we conclude that:

$$
\begin{aligned}
\omega \omega_{1}=b \underbrace{\omega^{\prime \prime} \cdot \omega_{1}^{\prime \prime}} a b a^{4(n-1)-3} & =b \cdot a^{3} \cdot a b a^{4(n-1)-3}=b^{2} a^{4} \cdot a^{4(n-1)-3} \\
& =a^{6} \cdot a^{4(n-1)-3}=a^{4(n-1)+3}=a^{3} .
\end{aligned}
$$

Hence $a^{3} S^{1}$ is the unique minimal right ideal of $S$.

Now, if we denote the minimal (two-sided) ideal of $S$ by $I$, then $a^{3} S^{1}=I=S^{1} a^{3}$. But $a^{3} S^{1} \cap S^{1} a^{3}=I$ is a group. Consequently, as a result of the Theorem 4 of [1], $I \cong G p\left(\pi_{1}\right)$.

To calculate the order of $S g\left(\pi_{1}\right)$, since $a^{2}=b^{2}=(a b)^{n}$, then every word that contains a subword $a b^{2}=b^{2} a=a^{3}$, is in $I$. So the only words that are not in $I$ are the followings: $a, b, a^{2}, a b, b a, a b a, b a b, \ldots,(a b)^{n-1} a, b(a b)^{n-1},(b a)^{n}$. All of these $4 n$ elements of $S$ are pairwise distinct and also each of them is alone in its $\mathscr{H}$-class and the number of $\mathscr{H}$-classes is $4 n+1$. So, $|S|=|I|+4 n$ or,

$$
\left|S g\left(\pi_{2}\right)\right|=\left|G p\left(\pi_{2}\right)\right|+4 n=4 n(n-1)+4 n=4 n^{2} .
$$


Trivially by the above discussion, all the Green's relations are equal in $S$, i.e.; $\mathscr{H}=\mathscr{L}=$ $\mathscr{R}=\mathscr{D}=\mathscr{J}$. This completes the proof.

Corollary 2.2. The semigroup $S$ where, $S=S g\left(\pi_{1}\right)$ has an unique idempotent $a^{4(n-1)}$.

Proof. By the Lemma 2.1, $a^{4(n-1)}$ is an idempotent of $S=S g\left(\pi_{1}\right)$. But by the Theorem A, it belongs to the unique minimal ideal of $S, I \cong G=G p\left(\pi_{2}\right)$, and so corresponds to the identity element of $G$. Also, none of the elements of $S-I$ is an idempotent of $S$.

\section{Proof of Theorem B}

An almost similar method to that of Theorem A will be used here.

Proof of Theorem B. Let $S=S g\left(\pi_{2}\right)$. We show that $S^{1} a^{l-1}$ and $a^{l-1} S^{1}$ are the unique left and unique right minimal ideals of $S$, respectively. As well as the proof of Theorem A we have to show, by induction on the length of $\omega$, that:

$$
\forall \omega \in\{a, b\}^{+}, \quad \exists \omega_{1} \in\{a, b\}^{+} ; \quad \omega_{1} \omega=a^{l-1}
$$

Let $l, m, n \geqslant 2$. If $|\omega|=1$ then $\omega \equiv a$ or $\omega \equiv b$. If $\omega \equiv a$ (in $S$ ), by setting $\omega_{1} \equiv b(a b)^{n-1} a^{l-1}$ we get:

$$
\begin{aligned}
\omega_{1} \omega & =b(a b)^{n-1} a^{l-1} \cdot a=b(a b)^{n-1} a^{l}=a^{l} b(a b)^{n-1} \\
& =a^{l-2} a \cdot a b(a b)^{n-1}=a^{l-2} a \cdot(a b)^{n}=a^{l-2} a=a^{l-1} .
\end{aligned}
$$

If $\omega \equiv b$ (in $S$ ), by putting $\omega_{1} \equiv a^{l-1}(a b)^{n-1} a$, we get:

$$
\omega_{1} \omega=a^{l-1}(a b)^{n-1} a \cdot b=a^{l-1}(a b)^{n}=a^{l-2} \cdot a(a b)^{n}=a^{l-2} \cdot a=a^{l-1} .
$$

Now, suppose that the assertion holds for all words with length $<k+1$, where $k \in \mathbb{N}$, and $\omega$ is a word of length $|\omega|=k+1$. If $\omega \equiv \omega^{\prime} a$, then by the induction hypothesis,

$$
\exists \omega_{1}^{\prime} \in\{a, b\}^{+} ; \quad \omega_{1}^{\prime} \omega^{\prime}=a^{l-1} .
$$

So, by defining $\omega_{1} \equiv b(a b)^{n-1} \omega_{1}^{\prime}$, we conclude that

$$
\begin{aligned}
& \omega_{1} \omega=b(a b)^{n-1} \underbrace{\omega_{1}^{\prime} \cdot \omega^{\prime}} a=b(a b)^{n-1} \cdot a^{l-1} \cdot a=a^{l} \cdot b(a b)^{n-1} \\
& =a^{l-2} \cdot a(a b)^{n} \quad=a^{l-2} \cdot a \quad=a^{l-1} .
\end{aligned}
$$

If $\omega \equiv \omega^{\prime \prime} b$ then,

$$
\exists \omega_{1}^{\prime \prime} \in\{a, b\}^{+} ; \quad \omega_{1}^{\prime \prime} \omega^{\prime \prime}=a^{l-1},
$$


and considering $\omega_{1} \equiv b(a b)^{n-2} a^{2} \omega_{1}^{\prime \prime}$ gives us

$$
\begin{aligned}
\omega_{1} \omega= & b(a b)^{n-2} a^{2} \underbrace{\omega_{1}^{\prime \prime} \cdot \omega^{\prime \prime}} b=b(a b)^{n-2} a^{2} \cdot a^{l-1} \cdot b \\
= & =a^{l-2} \cdot a(a b)^{n} \cdot b(a b)^{n-2} \cdot a b \\
& =a^{l-2} \cdot a \quad=a^{l-1} .
\end{aligned}
$$

Therefore, $S^{1} a^{l-1}=S a^{l-1} \cup\left\{a^{l-1}\right\}$ is the unique minimal left ideal of $S$.

To prove that $a^{l-1} S^{1}$ is the unique minimal right ideal of $S$ it suffices to show that

$$
\forall \omega \in\{a, b\}^{+}, \quad \exists \omega_{1} \in\{a, b\}^{+} ; \quad a^{l-1}=\omega \omega_{1} .
$$

If $|\omega|=1$, then $\omega \equiv a$ or $\omega \equiv b$. If $\omega \equiv a$, we set $\omega_{1} \equiv a^{l-2}(a b)^{n}, \quad(l>2)$ and we get the identity $\omega \omega_{1}=a \cdot a^{l-2}(a b)^{n}=a^{l-2} \cdot a(a b)^{b}=a^{l-1}$. (If $l=2$, we just set $\omega_{1} \equiv(a b)^{n}$ ). If $\omega \equiv b$, we set $\omega_{1} \equiv(a b)^{n-1} a^{l}$. Then,

$$
\omega \omega_{1}=b \cdot(a b)^{n-1} a^{l}=a^{l} \cdot b(a b)^{n-1}=a^{l-2} \cdot a(a b)^{n}=a^{l-1} .
$$

Letting $\omega=a \omega^{\prime}$ where $|\omega|=k+1$ and $\left|\omega^{\prime}\right|=k$ yields

$$
\exists \omega_{1}^{\prime} \in\{a, b\}^{+} ; \quad \omega^{\prime} \omega_{1}^{\prime}=a^{l-1} .
$$

So, by defining $\omega_{1} \equiv \omega_{1}^{\prime} b(a b)^{n-1}$, we get:

$$
\omega \omega_{1}=a \underbrace{\omega^{\prime} \cdot \omega_{1}^{\prime}} b(a b)^{n-1}=a \cdot a^{l-1} \cdot b(a b)^{n-1}=a^{l-2} \cdot a(a b)^{n}=a^{l-1} .
$$

In the case $\omega \equiv b \omega^{\prime \prime}$ where $\left|\omega^{\prime \prime}\right|=k<k+1$, the same argument gives us

$$
\exists \omega_{1}^{\prime \prime} \in\{a, b\}^{+} ; \quad \omega^{\prime \prime} \omega_{1}^{\prime \prime}=a^{l-1},
$$

and, by taking $\omega_{1} \equiv \omega_{1}^{\prime \prime} a(a b)^{n-1}$, we conclude that:

$$
\omega \omega_{1}=b \underbrace{\omega^{\prime \prime} \cdot \omega_{1}^{\prime \prime}} a(a b)^{n-1}=b \cdot a^{l-1} \cdot a(a b)^{n-1}=a^{l} b(a b)^{n-1}=a^{l-2} \cdot a(a b)^{n}=a^{l-1} .
$$

Hence $a^{l-1} S^{1}$ is the unique minimal right ideal of $S$.

Now, if we denote the minimal (two-sided) ideal of $S$ by $I$, then $a^{l-1} S^{1}=I=S^{1} a^{l-1}$. Consequently, $a^{l-1} S^{1} \cap S^{1} a^{l-1}=I$ is a group, indeed, the group $I \cong G p\left(\pi_{2}\right)$. To calculate the order of $S g\left(\pi_{2}\right)$, since $a^{2} \cdot b(a b)^{n-1}=a$, then $a \in a^{2} S^{1}$ and $a^{2} S^{1}=a S^{1}$ (for, $a^{2} \in a S^{1}$ ). This yields in turn $a^{i} S^{1}=a S^{1}$, for every positive integer $i$. Now, $a=a^{2} b(a b)^{n-1} \in a^{2} S^{1}=a^{l-1} S^{1}$ and so $a \in S^{1} a^{l-1}$. So $S^{1} a^{l}=S^{1} a^{l-1}=\cdots=S^{1} a$ and then, by $S^{1} b^{m}=S^{1} a^{l}$, we get

$$
S^{1} a^{i}=a^{i} S^{1}=I=S^{1} b^{m} \quad(\forall i=1, \ldots, l) .
$$


All of the elements of $S$ except $b, b^{2}, \ldots, b^{m-1}$ are in $I \cong G p\left(\pi_{2}\right)$ because they are pairwise distinct and

$$
\left\{b, b^{2}, \ldots, b^{m-1}\right\} \cap\left(\{a, b\}^{+} \backslash\left\{b^{i} \mid i \in \mathbb{N}\right\}\right)=\varnothing .
$$

Thus, $|S|=|I|+(m-1)$ or,

$$
\left|S g\left(\pi_{2}\right)\right|=\left|G p\left(\pi_{2}\right)\right|+(m-1)
$$

Also every $b^{i}(1 \leqslant i \leqslant m-1)$ is alone in its $\not{H}$-class and the [4] number of $\mathscr{H}$-classes is $(m-1)+1=m \quad\left(\left\{H_{a} \cong G p\left(\pi_{2}\right), H_{b}=\{b\}, H_{b^{2}}=\left\{b^{2}\right\}, \ldots, H_{b^{m-1}}=\left\{b^{m-1}\right\}\right\}\right)$. Trivially by the above discussion, all the Green's relations are equal in $S$, i.e.; $\mathscr{H}=\mathscr{L}=\mathscr{R}=\mathscr{D}=\mathscr{J}$. This completes the proof.

Corollary 3.1. In the semigroup $S$, where $S=S g\left(\pi_{2}\right)$ the element $(a b)^{n}$ is the unique idempotent of $S$.

Proof. Let $S=S g\left(\pi_{2}\right)$. Then by the first and second relations of $\pi_{2}$ we get the following equalities consecutively:

$$
\begin{aligned}
(a b)^{n} & =a b \cdot(a b)^{n-1} \\
& =a(a b)^{n} \cdot b(a b)^{n-1} \\
& =a(a b)(a b)^{n-1} \cdot b(a b)^{n-1} \\
& =a \cdot a(a b)^{n} \cdot b(a b)^{n-1} \cdot b(a b)^{n-1} \\
& =a^{2} \cdot a(a b)^{n} \cdot b(a b)^{n-1} \cdot b(a b)^{n-1} \cdot b(a b)^{n-1} \\
& =a^{3} \cdot(a b)^{n} \cdot\left(b(a b)^{n-1}\right)^{3} \\
& =\vdots \quad \vdots \quad \vdots \\
& =a^{l} \cdot(a b)^{n} \cdot\left(b(a b)^{n-1}\right)^{l} \\
& =(a b)^{n} \cdot a^{l} \cdot\left(b(a b)^{n-1}\right)^{l} \\
& =(a b)^{n} \cdot a^{l-1} \cdot a\left(b(a b)^{n-1}\right)\left(b(a b)^{n-1}\right)^{l-1} \\
& =(a b)^{n} \cdot a^{l-1} \cdot(a b)^{n}\left(b(a b)^{n-1}\right)^{l-1} \\
& =(a b)^{n} \cdot a^{l-2} \cdot a(a b)^{n} \cdot\left(b(a b)^{n-1}\right)^{l-1} \\
& =(a b)^{n} \cdot a^{l-2} \cdot a \cdot\left(b(a b)^{n-1}\right)^{\prime}\left(b(a b)^{n-1}\right)^{l-2} \\
& =(a b)^{n} \cdot a^{l-2} \cdot(a b)^{n} \cdot\left(b(a b)^{n-1}\right)^{l-2} \\
& =(a b)^{n} \cdot a^{l-3} \cdot a(a b)^{n} \cdot\left(b(a b)^{n-1}\right)^{l-2} \\
& =(a b)^{n} \cdot a^{l-3} \cdot a \cdot\left(b(a b)^{n-1}\right)^{l-2} \\
& =\vdots \quad \vdots \quad \quad \quad \vdots \\
& =(a b)^{n} \cdot a \cdot a(a b)^{n} \cdot\left(b(a b)^{n-1}\right)^{2} \\
& =(a b)^{n} \cdot a \cdot a \cdot b(a b)^{n-1} \cdot b(a b)^{n-1} \\
& =(a b)^{n} \cdot a(a b)^{n} \cdot b(a b)^{n-1} \\
& =(a b)^{n} \cdot a \cdot b(a b)^{n-1}=(a b)^{n} \cdot(a b)^{n} .
\end{aligned}
$$


Now, by the Theorem B, $(a b)^{n}$ belongs to the unique minimal ideal of $S=S g\left(\pi_{2}\right)$ which is a group and so it must be unique corresponding to identity element of $G=G p\left(\pi_{2}\right)$. Also, none of the elements of $S-I=\left\{b, b^{2}, \ldots, b^{m-1}\right\}$ is an [4] idempotent of $S$.

Remarks 3.2. We have obviously:

$$
\operatorname{Mon}\left(\pi_{1}\right) \cong S g\left(\pi_{1}\right) \cup\{1\} .
$$

Also, with a similar method as in proof of Theorem B, we can show that if

$$
\pi=\left\langle a, b, c \mid a^{l}=b^{m}=c^{n}, a b c=1\right\rangle,
$$

then $\operatorname{Mon}(\pi)$ is a group and $\operatorname{Mon}(\pi) \cong G p(\pi)$. On the other hand, if we let

$$
\begin{aligned}
& \pi_{3}=\left\langle a, b \mid a^{m}=b^{l},(a b)^{n} b=b\right\rangle, \\
& \pi_{4}=\left\langle a, b \mid a^{l}=b^{m},(a b)^{n} a=a\right\rangle, \\
& \pi_{5}=\left\langle a, b \mid a^{m}=b^{l}, b(a b)^{n}=b\right\rangle,
\end{aligned}
$$

then the groups presented by $\pi_{i},(i=2,3,4,5)$, are all isomorphic, but the semigroups presented by $\pi_{2}$ and $\pi_{3}$ are anti-isomorphic. While the anti-isomorphic semigroups presented by $\pi_{4}$ and $\pi_{5}$ are always infinite (for every values of $l, m, n>1$ ).

\section{References}

[1] C. M. Campbell, E. F. Robertson, N. Ruskuc and R. M. Thomas, Semigroup and group presentations, Bull. London Math. Soc., 27 (1995), 46-50.

[2] C. M. Campbell, J. D. Mitchell and N. Ruskuc, Comparing semigroup and monoid presentations for finite monoids, Monatsh. Math., 134 (2002), 287-293.

[3] C. M. Campbell, J. D. Mitchell and N. Ruskuc, On defining groups efficiently without using inverses, Math. Proc. Cambridge Philos. Soc., 133 (2002), 31-36.

[4] C. M. Campbell, E. F. Robertson, N. Ruskuc, R. M. Thomas and Y. Ünlü, Certain one-relator products of semigroups, Comm. Algebra, 23(14) (1995), 5207-5219.

[5] C. M. Campbell, E. F. Robertson, N. Ruskuc and R. M. Thomas, Fibonacci semigroups, J. Pure Appl. Algebra, 94 (1994), 49-57.

[6] C. M. Campbell, E. F. Robertson, and R.M. Thomas, On subsemigroups of finitely presented semigroups, J. Algebra, 180 (1996), 1-21.

[7] C. M. Campbell and P. P. Campbell, The Fibonacci lengths of binary polyhedral groups and related groups, Applications of Fibonacci Numbers, 10 , Kluwer, (Dordrecht, 2006), 83-91.

[8] C. M. Campbell, E. F. Robertson and R. M. Thomas, (2,n)-Semigroups, Technical report No. CSD-51, University of Leicester, 1992.

[9] H.S.M. Coxeter and W.O.J. Moser, Generators and Relations for Discrete Groups, 4th edition, Springer, Berlin, 1984.

[10] The GAP Group, GAP - Groups, Algorithms and Programming, Version 4.4 Aachen, St Andrews, 2006. (http://www.gap-system.org) 
[11] J. Howie, V. Metaftsis and R.M. Thomas, Triangle groups and their generalisations, in: Groups-Korea' 94 , eds. A.C. Kim and D.L. Johnson, Walter de Gruyter, Berlin, 1995, 135-147.

[12] D.L. Johnson, Presentations of Groups, Cambridge Unversity Press, (1997).

[13] E.F. Robertson and Y. Ünlü, On semigroup presentations, Proc. Edinburgh Math. Soc., 36 (1993), 55-68.

[14] N. Ruskuc, Semigroup presentations, Ph.D. Thesis, University of St. Andrews (1995).

[15] J. Wiegold, On some groups with trivial multiplicator, Bull. Austral. Math. Soc., 40 (1989), 331-332.

${ }^{1}$ Department of Mathematics, Tabriz Branch, Islamic Azad University, P. O. Box 1655, Tabriz, Iran.

E-mail: k_ahmadi@iaut.ac.ir; kdelir@gmail.com

${ }^{2}$ Mathematics Department, Science and Research Branch, Islamic Azad University, Tehran, P.O. Box 14515/1775, Iran.

E-mail: doostih@saba.tmu.ac.ir

${ }^{3}$ Department of Mathematics, Tabriz Branch, Islamic Azad University, P. O. Box 1655, Tabriz, Iran.

E-mail: gholami@iaut.ac.ir; rahmat_golamie@yahoo.com 